\title{
THE ANALYSIS OF THE CHARACTERS OF THE LIE REPRESENTATIONS OF THE GENERAL LINEAR GROUP
}

H. O. FOULKES

1. If a free Lie ring has $n$ generators and $A$ is a nonsingular $n \times n$ matrix of complex elements, then when the generators undergo a linear transformation of matrix $A$, the module of all forms of degree $m$ in the generators is mapped into itself by a linear transformation of matrix $L_{m}(A)$ on a set of basis elements. The mapping $A \rightarrow L_{m}(A)$ is a representation of the full linear group known as the $m$ th Lie representation [1]. The character of this representation has been shown [2] to be $\gamma_{m}=m^{-1} \sum_{d \mid m} \mu(d) s_{d}^{m / d}$, where $\mu(k)$ is the Möbius function of the integer $k$, and $s_{r}$ is the sum of the $r$ th powers of the eigenvalues of $A$. The decomposition of the $m$ th Lie representation into its irreducible constituents is in exact correspondence with the analysis of the symmetric function $\gamma_{m}$ into Schur functions. When $m$ is prime there is a simple rule for the coefficient $\alpha_{\lambda}$ of any $S$-function $\{\lambda\}$ in $\gamma_{m},[2]$, but there is no such rule when $m$ is composite. Since $s_{d}^{m / d}$ $=\sum_{\lambda} \chi_{d^{m / d}}^{\lambda}\{\lambda\}$, where $\chi_{\rho}^{\lambda}$ is the irreducible character of the class $(\rho)$ of the symmetric group $\mathfrak{S}_{m}$ corresponding to the partition $(\lambda)$ of $m$, it follows that $[10]$,

$$
\alpha_{\lambda}=\frac{1}{m} \sum_{d \mid m} \mu(d) \chi_{d^{m / d}}^{\lambda} .
$$

The calculation of $\alpha_{\lambda}$ thus reduces to the calculation of characters of the form $\chi_{d^{m / d} \text {. }}^{\lambda}$

It is the purpose of this note to suggest, in $\$ 2$, a method of calculation of these characters, and, in $\$ 3$, to indicate some relations satisfied by the $\alpha_{\lambda}$.

2. The well known formulae for $\chi_{1^{\prime}}^{\lambda}$ are

$$
{\chi 1^{m}}^{\lambda}=\frac{m ! \prod_{r<s}\left(\lambda_{r}-\lambda_{s}-r+s\right)}{\prod_{r}\left(\lambda_{r}+p-r\right) !}
$$

where $p=$ number of parts in $(\lambda)$;

$$
\chi_{1^{m}}^{\lambda}=\frac{m !}{\prod_{r, s}\left(\lambda_{r}+\tilde{\lambda}_{s}-r-s+1\right)},
$$

Received by the editors July 18, 1958 and, in revised form, October 8, 1958. 
where $(\tilde{\lambda})=\left(\tilde{\lambda}_{1}, \tilde{\lambda}_{2}, \cdots\right)$ is the partition conjugate to $(\lambda)$;

$$
\chi_{1^{m}}^{\lambda}=\frac{m !}{H_{\lambda}}
$$

where $H_{\lambda}$ is the product of the hook-lengths $h_{i j}$ in the hook-graph of ( $\lambda$ ) [3]. These give the coefficient of $\{\lambda\}$ in $s_{1}^{m}$. An alternative method, which extends to $\chi_{r^{m / r}}^{\lambda}$, the coefficient of $\{\lambda\}$ in $s_{r}^{m / r}$, is by evaluation of certain determinants; $[4$, pp. 134-135; $]$. Thus to find $\chi_{1^{10}}^{531^{2}}$ we evaluate

$$
10 !\left|\begin{array}{cccc}
\frac{1}{5 !} & \frac{1}{6 !} & \frac{1}{7 !} & \frac{1}{8 !} \\
\frac{1}{2 !} & \frac{1}{3 !} & \frac{1}{4 !} & \frac{1}{5 !} \\
\cdot & 1 & \frac{1}{1 !} & \frac{1}{2 !} \\
\cdot & \cdot & 1 & \frac{1}{1 !}
\end{array}\right|, \text { or preferably } 10 !\left|\begin{array}{cc}
\frac{35}{8 !} & \frac{4}{5 !} \\
\frac{1}{5 !} & \frac{1}{2 !}
\end{array}\right|
$$

obtained respectively from

$$
\left\{531^{2}\right\}=\left|\begin{array}{cccc}
\{5\} & \{6\} & \{7\} & \{8\} \\
\{2\} & \{3\} & \{4\} & \{5\} \\
\cdot & \{0\} & \{1\} & \{2\} \\
\cdot & \cdot & \{0\} & \{1\}
\end{array}\right| \text { and }\left\{531^{2}\right\}=\left|\begin{array}{cc}
\left\{51^{3}\right\} & \left\{21^{3}\right\} \\
\{5\} & \{2\}
\end{array}\right|,
$$

[6] giving 567. To find the coefficient of $\left\{531^{2}\right\}$ in $s_{r}^{10 / r}$ we replace every $\{k\}$ in the first of these determinants by zero if $k$ is not a multiple of $r$, and by $1 /(k / r)$ ! if it is. Multiplying the determinant by $(10 / r)$ ! gives the required coefficient. Thus the coefficients of $\left\{531^{2}\right\}$ in $s_{2}^{5}, s_{5}^{2}, s_{10}$ are respectively

$$
5 !\left|\begin{array}{cccc}
\cdot & \frac{1}{3 !} & \cdot & \frac{1}{4 !} \\
1 & \cdot & \frac{1}{2 !} & \cdot \\
\cdot & 1 & \cdot & 1 \\
\cdot & \cdot & 1 & \cdot
\end{array}\right|=15, \quad 2 !\left|\begin{array}{cccc}
1 & \cdot & \cdot & \cdot \\
\cdot & \cdot & \cdot & 1 \\
\cdot & 1 & \cdot & \cdot \\
\cdot & \cdot & 1 & .
\end{array}\right|=2 \text {, and zero, }
$$

giving the coefficient of $\left\{531^{2}\right\}$ in $\gamma_{10}$ as 55 . This was given as 53 by Thrall [1], but was later corrected by Brandt [2]. 
Justification of the above procedure is given conveniently by the use of the differential operator $D_{\lambda}$ obtained from $\{\lambda\}$ by replacing $s_{i}^{a}$ by $i^{a} \partial^{a} / \partial s_{i}^{a},[7]$. Thus the coefficient of $\{\lambda\}$ in $s_{1}^{m}$ is $D_{\lambda} s_{1}^{m}$, and if $(\lambda)=\left(\lambda_{1}, \lambda_{2}, \cdots, \lambda_{p}\right)$, the coefficient can be written as

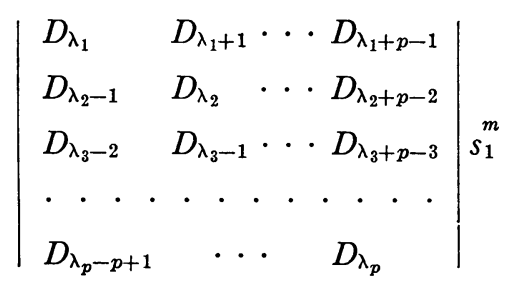

and the only effective part of each $D_{k}$ is $(k !)^{-1} \partial^{k} / \partial s_{1}^{k}$. Then in $D_{\lambda} s_{\tau}^{m / r}$ the only effective part of each $D_{k}$ is

$$
\frac{1}{k !} \frac{k !}{r^{k / r}(k / r) !} r^{k / r} \frac{\partial^{k / r}}{\partial s_{r}^{k / r}}=\frac{1}{(k / r) !} \frac{\partial^{k / r}}{\partial s_{r}^{k / r}}
$$

so that we get zero elements in the determinant when $k$ is not a multiple of $r$, and elements $1 /(k / r)$ ! when $k$ is a multiple of $r$.

3. We now list a number of results concerning the $\alpha_{\lambda}$.

I. When $m$ is prime, $\alpha_{\lambda}$ is the integer nearest to $m^{-1} \chi_{1^{m}}^{\lambda}$ [2].

II. $\alpha_{m}=0$ for $m>1,[10]$, and $\alpha_{1}^{m}=0$ for $m>2$.

These follow since $\alpha_{m}=m^{-1} \sum \mu(d)$, and $\alpha_{1}^{m}=m^{-1} \sum(-1)^{m+m / d} \mu(d)$. III. $\alpha_{m-1,1}=1$ for $m>1$, [10], and $\alpha_{21}{ }^{m-2}=1$ for $m>2$.

These follow since

$$
\chi_{1^{m}}^{m-1,1}=m-1, \quad \text { and } \quad \chi_{a^{b}}^{m-1,1}=-1 \text { for } a>1, b \geqq 1,
$$

$[8$, p. 137] and so

$$
\begin{aligned}
& \alpha_{m-1,1}=m^{-1}\left[m-1-\sum_{d \neq 1} \mu(d)\right]=1 \quad \text { for } m>1 \text {, } \\
& \alpha_{21^{m-2}}=m^{-1}\left[m-1-\sum_{d \neq 1}(-1)^{m+m / d} \mu(d)\right]=1, \quad \text { for } m>2 .
\end{aligned}
$$

IV. If $m$ is odd or a multiple of four, then $\alpha_{\lambda}=\alpha_{\tilde{\lambda}}$. It is well known that $\chi_{d^{m / d}}^{\lambda}=\chi_{d^{m / d}}^{\tilde{\lambda}}$ for odd values of $d$, and also for even $d$ whenever $m / d$ is even. This proves IV.

When $m$ is twice an odd integer, $\chi_{d^{m / d}}^{\lambda}=-\chi_{d^{m / d}}^{\tilde{\lambda}}$ for even $d$, and in this case $\gamma_{m}$, expressed in power sums, can be written as $P+Q$, where the coefficients of $\{\lambda\}$ and $\{\tilde{\lambda}\}$ are the same in $P$, but have opposite signs in $Q . P$ has all $s_{d}^{m / d}$ with odd $d$, and $Q$ has all $s_{d}^{m / d}$ with even $d$. There are certain partitions $(\lambda)$ for which the coefficient of $\{\lambda\}$ in $Q$ is zero, and for these $\alpha_{\lambda}=\alpha_{\tilde{\lambda}}$. 
The following three results are typical of many which can be obtained by equating appropriate coefficients when the right hand side of

$$
m^{-1} \sum_{d \mid m} \mu(d) s_{d}^{m / d}=\sum_{\lambda} \alpha_{\lambda}\{\lambda\}
$$

is expressed in terms of power sums by writing

$$
\{\lambda\}=(m !)^{-1} \sum_{\rho} h_{\rho} \chi_{\rho}^{\lambda} S_{\rho},
$$

where $h_{\rho}$ is the order of the class $(\rho)=1^{x_{1}} 2^{x_{2}} \cdots$ of $\subseteq_{m}$, and $S_{\rho}$ $=s_{1}^{x_{1}} s_{2}^{x_{2}} \ldots$. .

V. $\sum_{\lambda} \chi_{1^{m}}^{\lambda} \alpha_{\lambda}=(m-1)$ ! This is obtained by equating coefficients of $s_{1}^{m}$.

VI. $\sum_{\lambda=(m-r, 1 r)}(-1)^{r} \alpha_{\lambda}=\mu(m)$. Obtained by equating coefficients of $s_{m}$.

VII. If $S$-functions of ranks one and two are written respectively in Frobenius notation as

$$
\{\lambda\}=\left\{\begin{array}{l}
X_{r} \\
Y_{r}
\end{array}\right\} \quad \text { and } \quad\{\nu\}=\left\{\begin{array}{ll}
X_{t_{1}} & X_{t_{2}} \\
Y_{t_{1}} & Y_{t_{2}}
\end{array}\right\}
$$

and $a, b$ are any two unequal positive integers such that $a+b=m$, then $\sum_{\lambda} \theta_{r} \alpha_{\lambda}+\sum_{\nu} \phi_{t} \alpha_{\nu}=0$, where

$$
\begin{aligned}
\theta_{r} & =(-1)^{Y_{r}} & & \text { if } m>X_{r} \geqq a, \\
& =0 & & \text { if } a>X_{r} \geqq b, \\
& =(-1)^{Y_{r}+1} & & \text { if } b>X_{r} \geqq 0, \\
\phi_{t} & =(-1)^{Y_{t_{1}+Y_{t_{2}}}} & & \text { if } X_{t_{1}}+Y_{t_{1}}=a-1, \\
& =(-1)^{Y_{t_{1}+Y_{t_{2}+1}}} & & \text { if } X_{t_{1}}+Y_{t_{2}}=a-1, \\
& & \text { or if } X_{t_{2}}+Y_{t_{1}}=a-1, &
\end{aligned}
$$

This result follows by equating coefficients of $s_{a} s_{b}$, [9]. Other special relations of this kind may be obtained by equating coefficients of $s_{a} s_{b} s_{c}$, and of other terms. They depend on a knowledge of expressions such as $\theta_{r}, \phi_{t}$ above for the appropriate characteristics of $\mathfrak{S}_{m}$.

VIII. If $h_{d}$ is the order of the class $\left(d^{m / d}\right)$ of $\Xi_{m}$, then

$$
\sum_{\lambda} \alpha_{\lambda}^{2}=\frac{(m-1) !}{m} \sum_{d} \frac{1}{h_{d}},
$$

for square free values of $d$.

We have $D_{\gamma_{m}} \gamma_{m}=\sum_{\lambda} \alpha_{\lambda} D_{\lambda} \sum_{\lambda} \alpha_{\lambda}\{\lambda\}=\sum_{\lambda} \alpha_{\lambda}^{2}$. Also 


$$
\begin{aligned}
D_{\gamma_{m}} \gamma_{m} & =m^{-1}\left[\sum_{d} \mu(d) d^{m / d} \frac{\partial^{m / d}}{\partial s_{d}^{m / d}}\right] m^{-1} \sum_{d} \mu(d) s_{d}^{m / d} \\
& =\left(m^{2}\right)^{-1}\left[\sum(\mu(d))^{2} d^{m / d}(m / d) !\right] \\
& =\left(m^{2}\right)^{-1} \sum \frac{m !}{h_{d}}
\end{aligned}
$$

for square free values of $d$.

More explicit results may be written down when $m$ has some prescribed form. Thus when $m$ is a prime $p, \sum \alpha_{\lambda}^{2}=p^{-1}[1+(p-1) !]$, and when $m$ is the product of two distinct primes $p, q$,

$$
\sum \alpha_{\lambda}^{2}=\frac{1}{p q}\left[(p q-1) !+p^{q-1}(q-1) !+q^{p-1}(p-1) !+1\right] .
$$

When $m$ is not twice an odd number we have $\alpha_{\lambda}=\alpha_{\lambda}$ by IV. But if $m=2(2 k+1)$, then by evaluating $D_{\gamma_{m}} \tilde{\gamma}_{m}$ in two ways, we obtain a further result;

IX. If $m$ is twice an odd number, then $\sum_{\lambda} \alpha_{\lambda} \alpha_{\lambda}=(m-1) ! / m$ $\cdot \sum_{d}(-1)^{d+1}\left(h_{d}\right)^{-1}$ for square free values of $d$.

Some results on the characters of the Lie representations in the special case when the number of generators is two have been given in a recent paper by Davis [10].

I am indebted to the referee for his comments.

\section{REFERENCES}

1. R. M. Thrall, On symmetrized Kronecker powers and the structure of the free Lie ring, Amer. J. Math. vol. 64 (1942) pp. 371-388.

2. A. J. Brandt, The free Lie ring and Lie representations of the full linear group, Trans. Amer. Math. Soc. vol. 56 (1944) pp. 528-536.

3. J. S. Frame, G. de B. Robinson, and R. M. Thrall, The hook graphs of the symmetric group, Canad. J. Math. vol. 6 (1954) pp. 316-324.

4. F. D. Murnaghan, Theory of group representations, Baltimore, 1938.

5. H. O. Foulkes, A note on S-functions, Quart. J. Math. Oxford Ser. (2) vol. 20 (1949) pp. 190-192.

6. - Reduced determinantal forms for S-functions, Quart. J. Math. Oxford Ser. (2) vol. 2 (1951) pp. 67-73.

7. —_ Differential operators associated with S-functions, J. London Math. Soc. vol. 24 (1949) pp. 136-143. 1940.

8. D. E. Littlewood, Group characters and matrix representations of groups, Oxford,

9. H. O. Foulkes, Monomial symmetric functions, $S$-functions, and group characters, Proc. London Math. Soc. vol. 3 no. 2 (1952) pp. 45-59.

10. R. L. Davis, A special formula for the Lie character, Canad. J. Math. vol. 10 (1958) pp. 33-38.

University College, Swansea, Wales 